\title{
Clinical significance of an ultrafast alkaline phosphatase isoenzyme
}

\author{
JOHN KOETT, JAMES HOWELL, AND PAUL L. WOLF \\ From the Department of Laboratory Medicine, Naval Regional Medical Center, San Diego, \\ California 92134, and the Department of Pathology, University of California, La Jolla, \\ California 92093, USA
}

SUMMARY We have studied five patients who have exhibited an unusual alkaline phosphatase isoenzyme (ALP EC 3.1.3.1) migrating in an ultrafast position electrophoretically on cellulose acetate. This ALP isoenzyme has been identified in patients with benign and malignant liver diseases. In addition, a number of these patients exhibited a regular ALP liver isoenzyme and a fast (preliver) ALP isoenzyme in conjunction with the ultrafast ALP liver isoenzyme.

This paper reports the finding of a unique isoenzyme of ALP which has not been observed previously on review of the literature. Since this isoenzyme migrates towards the anode and is faster than the isoenzyme described by Fritsche and Adams-Park $(1972 ; 1974)$, we have chosen to call it the ultrafast liver isoenzyme of ALP. Previous investigators have identified a regular liver isoenzyme and an abnormally anodally migrating enzyme referred to as preliver or fast liver. In contrast to these ALP isoenzymes, which we have likewise observed, the ultrafast isoenzyme migrates faster towards the anode than the other two liver isoenzymes in the area corresponding to albumin on cellulose acetate electrophoretic media.

\section{Material and methods}

Patients' sera were selected on the basis of an elevated ALP and were electrophoresed immediately or stored at $-70^{\circ} \mathrm{C}$ until the day of electrophoresis. Clinical information was correlated with the laboratory findings.

The isoenzymes of alkaline phosphatase were delineated using the Helena Laboratory alkaline phosphatase isoenzyme procedure, which entailed the use of Titan III-Iso cellulose acetate plates, $60 \times 75 \mathrm{~mm}$, Electro HR (barbital) buffer, and alpha naphthol ASMX phosphate reagent, which was subsequently coupled with the diazo compound, Fast Blue RR, yielding sharp blue visible bands. The

Received for publication 17 May 1979 developed plates were then scanned on an Auto Scanner Flur-Vis densitometer.

Identification of individual isoenzyme bands was made utilising reference alkaline phosphatase control sera supplied by the Helena Laboratory consisting of bovine intestinal and liver isoenzymes.

To determine if the ultrafast liver isoenzyme was an artifact of a complex bilirubin and albumin ando Fast Blue RR, a number of serum samples with: elevated serum bilirubin and alkaline phosphatase levels were electrophoresed and subsequently developed with ASMX (containing alpha naphthol phosphate) and Fast Blue RR or barbital buffer (pH 8.8 with Fast Blue RR only).

\section{Results}

We observed a fast liver fraction in conjunction with an ultrafast liver band in three patients with various diseases, including carcinoma of the head of the pancreas with metastasis to the liver (Fig. 2), ovarian carcinoma with suspected metastasis to the liver (Fig. 3), and breast carcinoma metastatic to the liver (Fig. 4). This unique ultrafast liver isoenzyme has also been observed in conjunction with a regular liver isoenzyme in the absence of the fast liver fraction (Figs 1, 5, 6). The representative combination of isoenzymes with correlated clinical histories are listed in Table 1. Representative densitometry tracings from cases 1 to 6 and cases $8,9,10$, and 14 are illustrated. In all patients there was an elevation of the serum ALP but not necessarily an elevation of the patient's bilirubin. The total ALP ranged from $95 \mathrm{U} / 1$ to approximately 


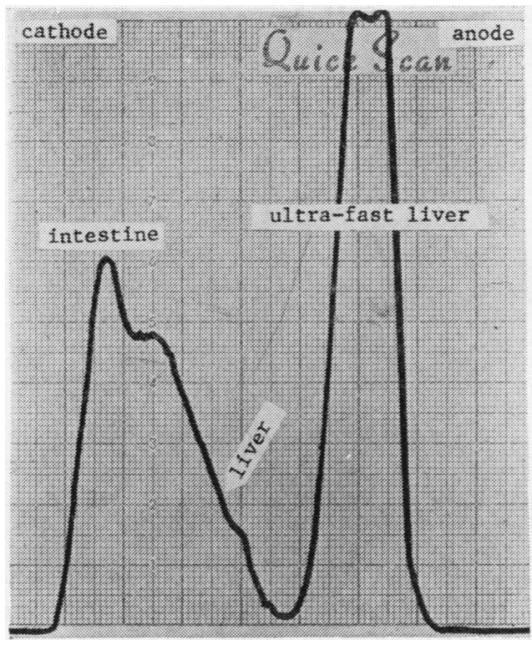

Fig. 1 Mrs GW, aged 61 with Laennec's cirrhosis.

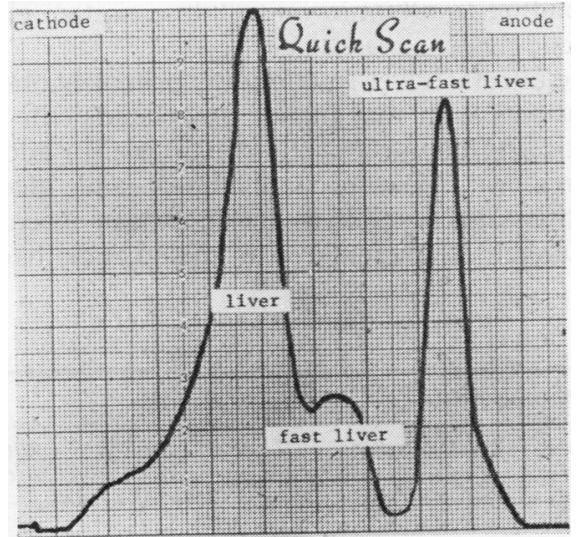

Fig. $2 L H$, a 72-year-old woman with carcinoma of the head of the pancreas/obstruction/metastasis to the liver.

$600 \mathrm{U} / 1$ as determined on the SMAC instrument (Technicon Inc, Tarrytown, New York). Low levels of total ALP did not appear to preclude the presence of the ultrafast liver isoenzyme ALP, as indicated in patient MD (Fig. 5) whose total activity was $95 \mathrm{U} / \mathrm{l}$. Multiple determinations of the isoenzyme electrophoretic pattern did not alter the presence or location of the isoenzyme even after storage at $-70^{\circ} \mathrm{C}$ for approximately one to two weeks. Follow-up studies on the ultrafast fraction in patients with chronic disease continued to demonstrate the presence of this ultrafast fraction, as was the case with patient GW (Fig. 1) after approximately a one-month lapse between analyses. In contrast,

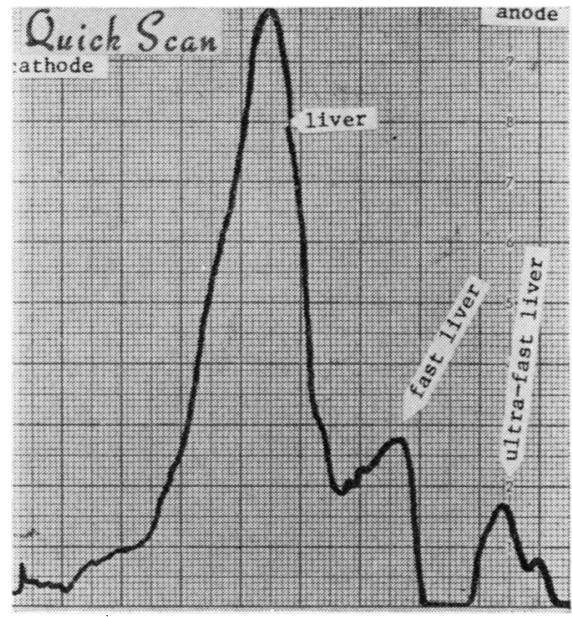

Fig. 3 Mrs HC aged 60 with ovarian carcinomal metastatic with ascites.

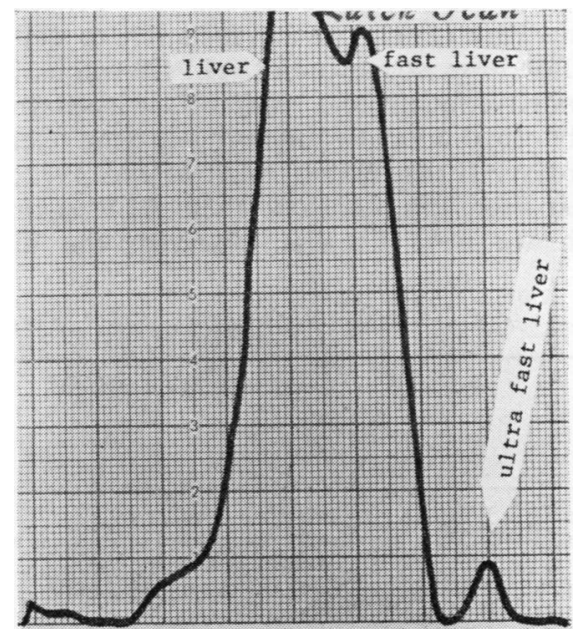

Fig. 4 Mrs MA aged 60 with metastatic breast carcinoma to the liver.

patient HR (case 7), listed in Table 1 with a transient viral syndrome, demonstrated an elevated ALP and LD, which on fractionation of the former enzyme demonstrated a fast liver isoenzyme that disappeared on resolution of the viraemia.

\section{Discussion}

This paper reports the findings of a unique isoenzyme of ALP which, on review of the literature, has not been observed previously. Since this iso- 


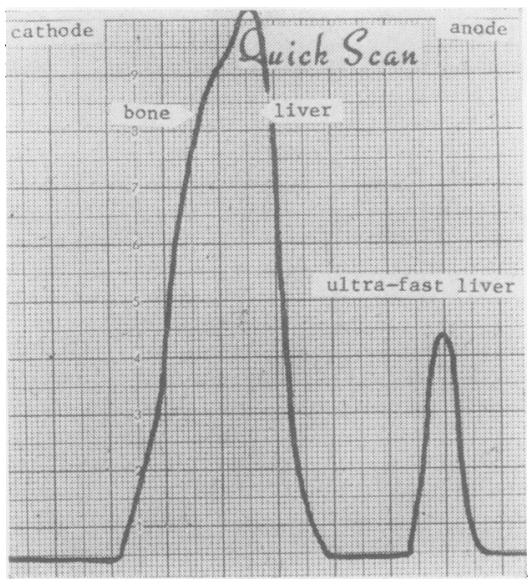

Fig. 5 Mrs MD aged 55 with multiple areas of increased isotope uptake on bone scan and an abnormal LS-scan consistent with alcoholic cirrhosis.

enzyme migrates towards the anode and is faster than the isoenzyme described by Fritsche and Adams-Park (1972), we have chosen to call it the ultrafast liver isoenzyme of ALP. Previous investigators have identified a regular liver isoenzyme and an abnormal anodally migrating enzyme referred to as preliver (Fritsche and Adams-Park, 1972; 1974) or fast liver. In contrast to these ALP isoenzymes, which we have likewise observed, the ultrafast

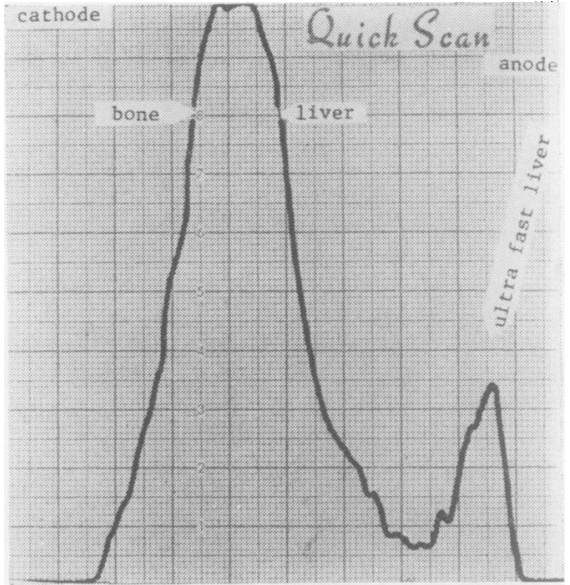

Fig. 6 Mrs $Z W$ aged 55 with abnormal liver function tests, probably induced by allopurinol and/or colchicine

isoenzyme migrates faster towards the anode than the other two liver isoenzymes in the area corresponding to albumin on cellulose acetate electrophoretic media.

The ultrafast liver fraction was primarily observed in patients with malignancy associated with extrahepatic and/or intrahepatic cholestasis. In addition patients with Laennec's cirrhosis and alcoholic hepatitis also exhibited the ultrafast isoenzyme. A number of patients with malignancy had the

Table 1 Correlation of clinical findings and ALP isoenzymes

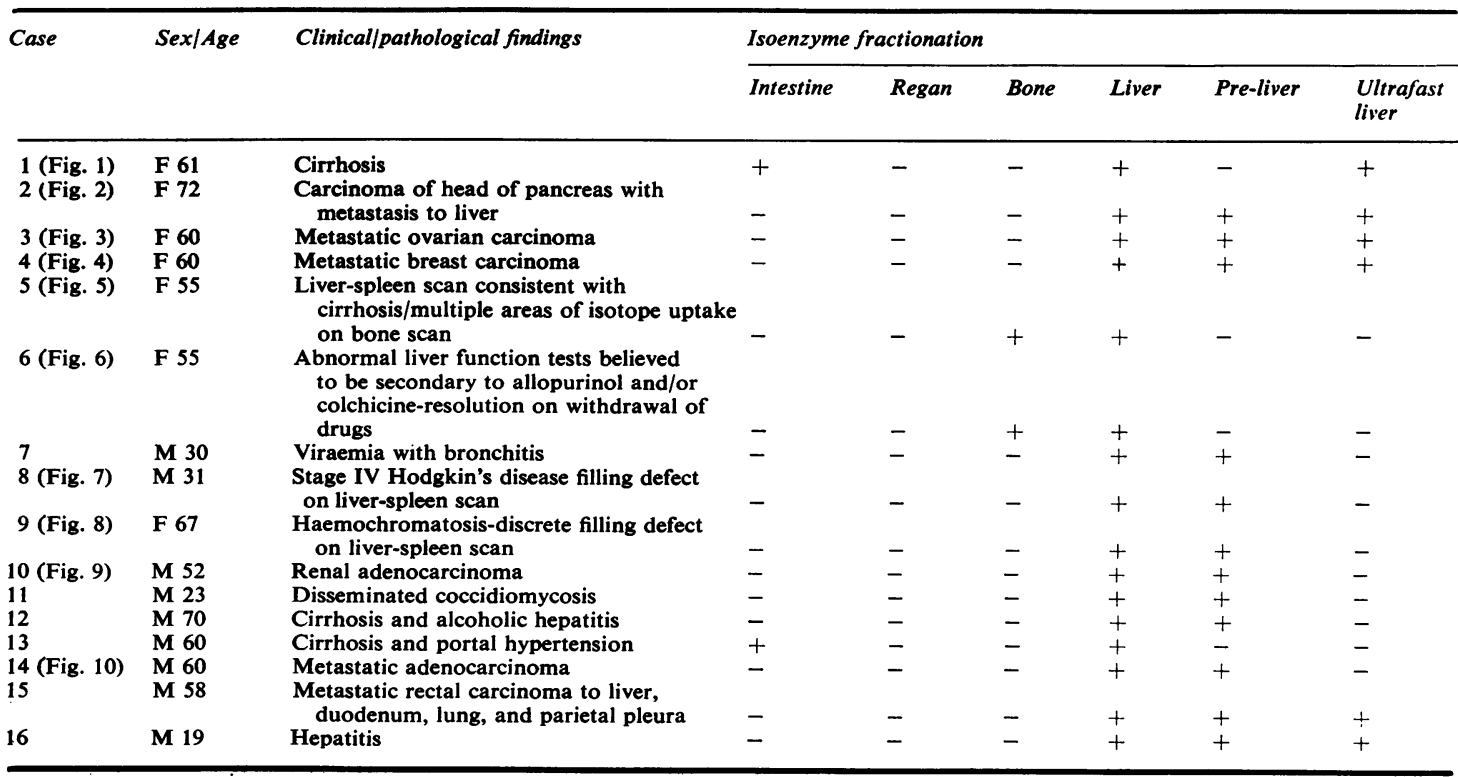


simultaneous presence of three liver isoenzymes: the regular enzyme, fast liver, and the ultrafast liver isoenzyme. This pattern did not occur in patients with cirrhosis or alcoholic hepatitis. One patient had an ultrafast liver fraction caused by a drug-induced hepatitis (case 6; Fig. 6). We have also observed a fast liver fraction associated with Hodgkin's disease causing a large filling defect of the liver on liverspleen scan (case 8; Fig. 7). We have also seen this similar isoenzyme pattern in one patient with haemochromatosis (case 9; Fig. 8) and renal cell adenocarcinoma (case 10; Fig. 9), and in another patient with disseminated coccidioidomycosis (case 11), and adenocarcinoma of lung (case 14; Fig. 10).

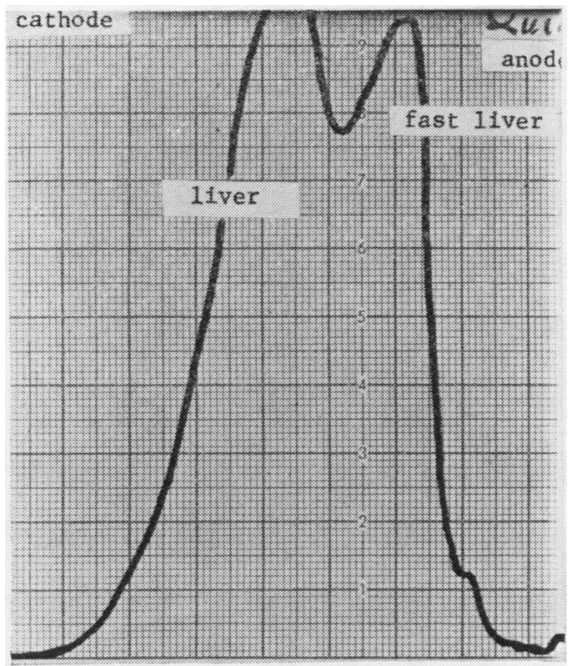

Fig. $7 \quad H G, 31$-year-old man with stage IV-B Hodgkin's disease/superior-portal filling defect on $L S$-scan.

We have recently become disenchanted with the heat stability test for ALP since we do not feel this test yields definitive, clinically useful information. Confusing results are obtained with the heat test owing to the presence of multiple isoenzymes of ALP exhibiting different heat inactivation attributes (Fishman, 1974; Whitby and Moss, 1975). We therefore have sought another modality to aid in the differential diagnosis of an elevated serum ALP. We chose the Helena Laboratory isoenzyme electrophoresis procedure on cellulose acetate membranes because of the ease of handling, stability of the supporting membrane and media, and the reproducibility of results. All these factors have made this procedure attractive for the laboratory worker in an extremely active clinical laboratory. In contrast, although acrylamide gel electrophoresis is more discriminating because of its molecular sieving action as well as its separation on the basis of charge, this medium requires preparation of individual components and polymerisation steps and in some cases pre-electrophoresis to remove any potential interfering substances that might inhibit enzymatic activity (Gordon, 1971; Emery and Dounce, 1955; Thines-Sempoux, 1973).

Previous investigators, using different separating media, have identified the presence of a fast liver band in patients with liver disease (Fritsche and Adams-Park, 1974; Afonja and Baron, 1974), and this band may represent the slow moving isoenzyme noted in polyacrylamide gel electrophoresis (Fritsche and Adams-Park, 1974; Price and Sammons, 1974). De Broe et al., 1975 found that the high molecular weight alkaline phosphatase with rapid migration on cellulose acetate electrophoresis corresponded to a membrane-vesicle fraction seen with electron microscopy in patients with cholestasis. The unique isoenzyme that we have observed may be a posttranslationally modified enzyme of the liver type associated with a lecithin-liver isoenzyme complex, which induces a change in its electrophoretic mobility, as described by Price and Sammons (1974) in obstructive liver disease. However, studies in our laboratory utilising Triton X-100 anionic detergent induced no appreciable change in the isoenzyme pattern previously noted. At the present time we are not able to identify the genesis of this newly described ALP isoenzyme. We are presently actively investigating the inhibitory effect of different amino acids as well as classical chemical agents utilised to disrupt hydrogen bonding to characterise further

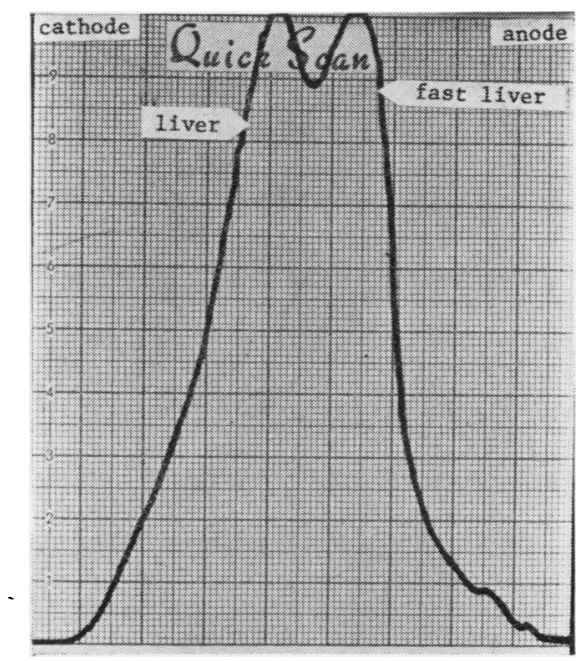

Fig. 8 Mrs MJ aged 67 with biopsy-proven haemochromatosis/filling defect on liver/spleen scan. 


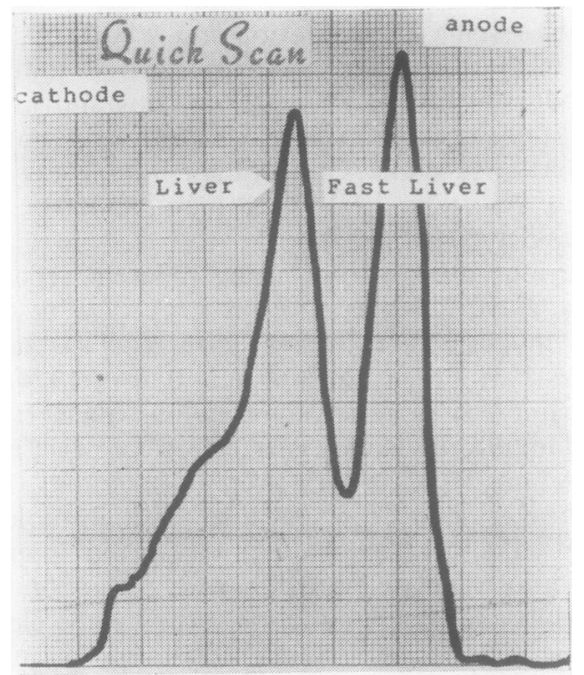

Fig. $9 V T$, a 52-year-old man with metastatic renal carcinoma.

the ultrafast isoenzyme. Moreover, we are investigating the effect of enzymatic degradation of the new isoenzyme in an attempt to define its reactivity in the absence of potentially critical carbohydrate moieties.

Previous reports have identified three isoenzymes of alkaline phosphatase in patients with cirrhosis (Fishman, 1974). They include the regular liver, bone, and intestinal isoenzyme. The elevation of the intestinal isoenzyme has been noted by us (Table 1) and may be related to anoxic injury to the gastrointestinal tract induced by portal hypertension. The elevated bone fraction has been thought to be secondary to a defect or decreased activity in the 25-hydroxylase enzyme of the liver in cirrhosis, which converts cholecalciferol to its 25 -hydroxylated product, which is the precursor to the active metabolite 1,25 di-hydroxycholecalciferol via the one hydroxylase of the kidney. This latter vitamin D derivative has been shown to be intimately involved with calcium absorption from the gastrointestinal tract. With inadequate physiological quantities, a relative hypocalcaemia ensues with secondary activation of PTH secretion by the parathyroid glands, thereby increasing osteoblastic and osteoclastic activity with resultant elevation of the bone isoenzyme of ALP. We have seen this isoenzyme pattern in a number of patients with alcoholic cirrhosis.

Recently, Ehrmeyer et al. (1978) demonstrated the

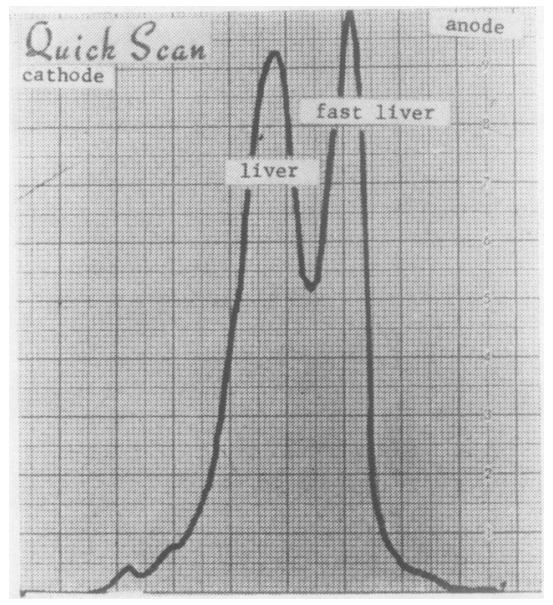

Fig. $10 L F$, a 60-year-old man with adenocarcinoma of the lung metastatic to brain and skeleton. Liver/spleen scan normal.

Table 2 Correlation of bilirubin and ALP isoenzymes

\begin{tabular}{|c|c|c|c|c|c|}
\hline & Liver & Pre-liver & UF liver & $\begin{array}{r}\text { Bilirubin } \\
(m g / d l)\end{array}$ & Total $A L P(U / f l)$ \\
\hline \multicolumn{6}{|l|}{ (Case 16, Table 1) } \\
\hline Alpha naphthol phosphate + Fast Blue RR & + & + & + & $>20$ & 247 \\
\hline $\begin{array}{l}\text { Fast Blue RR only } \\
\text { (Case } 15 \text { Table 1) }\end{array}$ & - & - & + & & \\
\hline Alpha naphthol phosphate + Fast Blue RR & + & + & + & $12 \cdot 9$ & 496 \\
\hline Fast Blue RR only & - & - & + & & \\
\hline \multicolumn{6}{|l|}{ (Random specimen) } \\
\hline Alpha naphthol phosphate + Fast Blue RR & + & - & + & $9 \cdot 2$ & 77 \\
\hline Fast Blue RR & - & - & - & & \\
\hline \multicolumn{6}{|l|}{ (Random specimen) } \\
\hline Alpha naphthol phosphate + Fast Blue RR & + & - & + & $6 \cdot 2$ & 62 \\
\hline Fast Blue RR only & - & - & - & & \\
\hline \multicolumn{6}{|l|}{ (Random specimen) } \\
\hline Alpha naphthol phosphate + Fast Blue RR & + & - & + & $3 \cdot 7$ & 70 \\
\hline Fasț Blue RR & - & - & - & & \\
\hline
\end{tabular}

$\mathrm{UF}=$ ultrafast 
presence of a fast-liver fraction referred to as fast, homoarginine sensitive alkaline phosphatase that migrates in the $\alpha_{1}$ serum protein position on cellulose acetate media in patients with various carcinomas. Unlike Ehrmeyer's findings, our ultrafast liver fraction migrates anodally to his identified band. However, in concert with Ehrmeyer's findings, we did identify a preliver or fast-liver fraction in patients with cancer.

The ultrafast liver band has been attributed to an artifact caused by a bilirubin albumin complex and Fast Blue RR utilised in the electrophoretic technique (Hardin et al., 1978). However, another publication (Tsung, 1978) disputed Hardin's conclusion and described two patients with normal serum bilirubin levels, one with granulomatous hepatitis and the other with carcinoma of the pancreas metastatic to the liver who demonstrated an ultrafast alkaline phosphatase band on electrophoresis. Because of these reports, we chose random serum samples from patients with variably elevated serum bilirubin and alkaline phosphatase.

To determine if the ultrafast liver isoenzyme was an artifact of a complex bilirubin and albumin and Fast Blue RR, a number of serum samples with elevated serum bilirubin and alkaline phosphatase

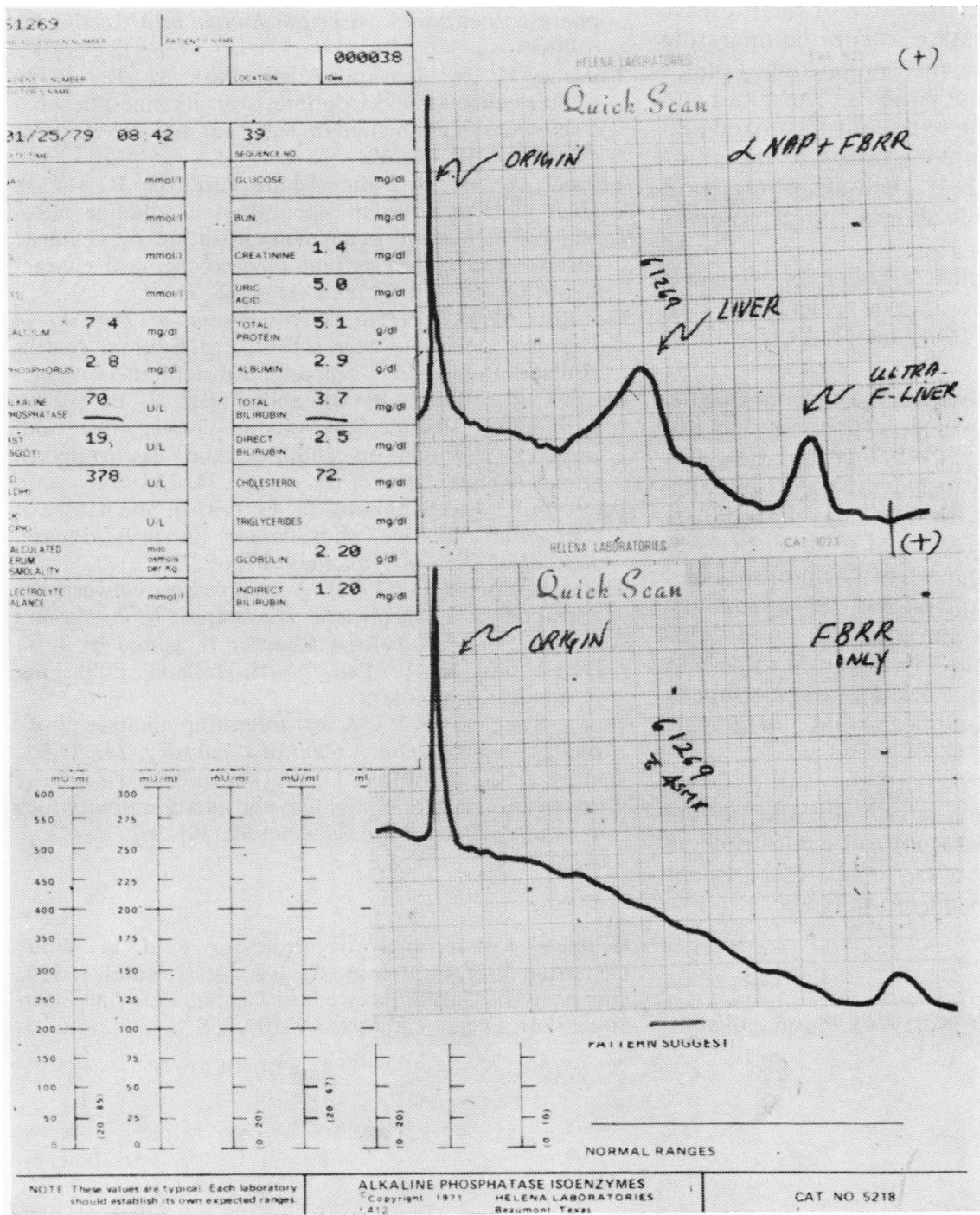

Fig. 11 Electrophoretic strip, stained with Fast Blue RR only, demonstrates a small band displaced anodally relative to the ultrafast liver isoenzyme band. 
levels were electrophoresed and subsequently developed with ASMX (containing alpha naphthol phosphate) and Fast Blue RR or barbital buffer (pH 8.8 with Fast Blue RR only). Two of the specimens studied were from patients listed in Table 1 (cases 15 and 16) and are indicated in Table 2. The remaining specimens were chosen randomly without regard to their clinical histories and solely on the basis of an elevated serum bilirubin.

The results listed in Table 2 (patients 15 and 16) demonstrate that the 'ultrafast' liver isoenzyme in these two patients is really an artifactual band produced by the interaction of bilirubin, albumin, and Fast Blue RR, as suggested by Hardin. However, the results in patients 3 to 5 are not consistent with Hardin's findings and are suggestive of the presence of a true alkaline phosphatase isoenzyme migrating as an ultrafast liver isoenzyme, supporting Tsung's report. In addition, in these patients ( 3 to 5) a band that was displaced anodally to the ultrafast band and very small in amplitude in comparison to the ultrafast band was observed. This small absorbing band probably represents a bilirubin albumin complex (Fig. 11).

In conclusion, the ultrafast alkaline phosphatase isoenzyme appears to be a true isoenzyme and associated most commonly with neoplastic disease of the liver.

In summary, we have described a new isoenzyme of alkaline phosphatase, which surprisingly has not been reported previously. Whether or not this isoenzyme is only demonstrated utilising the alpha naphthol substrate of the Helena Laboratory procedure has not been elucidated to date. Perhaps other substrates such as thymolphthalein phosphate, beta glycerol phosphate, or indoxyl phosphate will likewise react with this enzyme. We hope this paper will encourage other investigators to help to identify and corroborate our findings and attempt to assign this isoenzyme clinical and diagnostic relevancy.

The opinions or assertions herein are the private views of the authors and are not to be construed as official or as reflecting the views of the Department of Defense or the Department of the Navy.

\section{References}

Afonja, A. D., and Baron, D. N. (1974). Plasma alkaline phosphatase isoenzymes in hepatobiliary disease. Journal of Clinical Pathology, 27, 916-920.

De Broe, M. E., Borgers, M., and Wieme, R. J. (1975). The separation and characterization of liver plasma membrane fragments circulating in the blood of patients with cholestasis. Clinica Chimica Acta, 59, 369-372.

Ehrmeyer, S. L., Joiner, B. L., Kahan, L., Larson, F. C., and Metzenberg, R. L. (1978). A cancer-associated, fast, homoarginine-sensitive electrophoretic form of serum alkaline phosphatase. Cancer Research, 38, 599-601.

Emery, A. J., Jr., and Dounce, A. L. (1955). Intracellular distribution of alkaline phosphatase in rat liver cells. Journal of Biophysical and Biochemical Cytology, 1, 315-330.

Fishman, W. H. (1974). Perspectives on alkaline phosphatase isoenzymes. American Journal of Medicine, 56, 617-650.

Fritsche, H. A., Jr., and Adams-Park, H. R. (1972). Cellulose acetate electrophoresis of alkaline phosphatase isoenzymes in human serum and tissue. Clinical Chemistry, 18, 417-421.

Fritsche, H. A., Jr., and Adams-Park, H. R. (1974). High molecular weight isoenzymes of alkaline phosphatase in human serum demonstration by cellulose acetate electrophoresis and physico-chemical characterization. Clinica Chimica Acta, 52, 81-89.

Gordon, A. H. (1971). Electrophoresis of Proteins in Polyacrylamide and Starch Gels, 3rd Edition. NorthHolland Publishing Company, Amsterdam-London.

Hardin, E., Passey, R. B., and Fuller, J. B. (1978). Artifactual alkaline phosphatase isoenzyme band, caused by bilirubin, on cellulose acetate electro-pherograms. (Letter) Clinical Chemistry, 24, 178-179.

Price, C. P., and Sammons, H. G. (1974). The nature of the serum alkaline phosphatases in liver disease. Journal of Clinical Pathology, 27, 392-398.

Thines-Sempoux, D. (1973). A comparison between the lysosomal and the plasma membranes. In Lysosomes in Biology and Pathology, Chapter II, edited by J. T. Dingle and H. B. Fell. North-Holland Publishing Company, Amsterdam.

Tsung, Swei, H. (1978). A fast-migrating alkaline phosphatase band. (Letter.) Clinical Chemistry, 24, 2068.

Whitby, L. G., and Moss, D. W. (1975). Analysis of heat inactivation curves of alkaline phosphatase isoenzymes in serum. Clinica Chimica Acta, 59, 361-367.

Requests for reprints to: Professor Paul L. Wolf, Department of Pathology, University Hospital H-720, University of California Medical Center, 225 Dickinson Street, San Diego, California 92103, USA. 